\title{
Interior estimates for hypersurfaces moving by mean curvature
}

\author{
Klaus Ecker $^{1}$ and Gerhard Huisken ${ }^{2}$ \\ ${ }^{1}$ Department of Mathematics, The University of Melbourne, Parkville VIC 3052, Australia \\ ${ }^{2}$ Centre for Mathematical Analysis, Australian National University, GPO Box 4, \\ Canberra ACT 2601, Australia
}

Oblatum 19-X-1990

Let $\mathbf{F}_{t}: M^{n} \rightarrow R^{n+1}$ be a hypersurface moving by its mean curvature in Euclidean space. That is, $\mathbf{F}_{t}=\mathbf{F}(\cdot, t)$ is a one-parameter family of smooth immersions, with images $M_{t}=\mathbf{F}_{t}\left(M^{n}\right)$, satisfying the evolution equation

$$
\frac{d}{d t} \mathbf{F}(p, t)=\mathbf{H}(p, t), \quad p \in M^{n}, t>0 .
$$

Here $\mathbf{H}(p, t)=-H(p, t) \cdot v(p, t)$ is the mean curvature vector of $M_{t}$ at $\mathbf{F}(p, t)$ and $v$ denotes a choice of unit normal for $M_{t}$.

Mean curvature evolution of smooth hypersurfaces was studied previously under various global assumptions: It was shown in $[8,6]$ that compact convex surfaces in $R^{n+1}, n \geqq 2$ and embedded curves in the plane contract smoothly to a point. In [4] the authors characterized the longterm behaviour of entire graphs of controlled growth.

It is the aim of this paper to study the local properties of mean curvature flow and obtain regularity estimates which are interior both in space and time. Working in a region sufficiently small such that $M_{t}$ can be written as the graph of a function $w$ over some hyperplane, we use the gradient function $v$ $=\sqrt{1+|D w|^{2}}$ to obtain interior estimates for the second fundamental form $A$ and all its derivatives, compare Theorem 3.4. Under additional curvature assumptions on $M_{t}$ we furthermore obtain improved estimates with respect to time dependence for the derivatives of $A$ without having to assume that $M_{t}$ can be locally written as a graph. It is worth noting that all the interior estimates also apply to the case of minimal hypersurfaces as these are stationary solutions of (1), compare Remark 3.6.

An immediate consequence of the interior estimates is a shorttime existence result for the mean curvature flow of complete surfaces, assuming only a uniform local Lipschitz condition for the initial surface. In Sect. 4 we extend a method of Liao and Tam [16] to prove a general maximum principle for parabolic equations on such complete Riemannian manifolds with time-dependent metric. As an application of this maximum principle we obtain that nonnegative mean curvature is preserved by (1) under reasonably weak conditions. 
Finally, in Sect. 5 we use the interior estimates to deduce the somewhat surprising result that if $M_{0}$ is a locally Lipschitz continuous entire graph over $R^{n}$, then the mean curvature flow Eq. (1) has a smooth solution with initial data $M_{0}$ which exists for all times $t>0$. In particular, it is not necessary to make any assumption on the growth and the curvature of $M_{0}$ for $|x| \rightarrow \infty$.

\section{Preliminaries}

We will use the same notation as in [10] and [4], in particular we will often write $\mathbf{x}=\mathbf{F}(p, t)$ to denote the position of a point in $M_{t}$ and write $A=\left\{h_{i j}\right\}$ for the second fundamental form of $M_{t}$.

Furthermore, if $M_{t}$ is locally given as a graph over some hyperplane in $R^{n+1}$ such that $\langle v, \omega\rangle>0$ for some fixed vector $\omega \in R^{n+1},|\omega|=1$, we will consider the gradient function $v$ defined by

$$
v=\langle v, \omega\rangle^{-1} \text {. }
$$

With this notation we recall the following evolution equations from [10] and [4].

1.1 Lemma. If $M_{t}$ satisfies (1) we have
(i) $\left(\frac{d}{d t}-\Delta\right)|\mathbf{x}|^{2}=-2 n$,
(ii) $\left(\frac{d}{d t}-\Delta\right) u=0$, where $u=\langle\mathbf{x}, \omega\rangle$,
(iii) $\left(\frac{d}{d t}-\Delta\right) v=-|A|^{2} v-2 v^{-1}|\nabla v|^{2}$,
(iv) $\left(\frac{d}{d t}-\Delta\right) H=H|A|^{2}$,
(v) $\left(\frac{d}{d t}-\Delta\right)|A|^{2}=-2|\nabla A|^{2}+2|A|^{4}$,
(vi) $\left(\frac{d}{d t}-\Delta\right)\left|\nabla^{m} A\right|^{2} \leqq-2\left|\nabla^{m+1} A\right|^{2}+C(m, n) \sum_{i+j+k=m}\left|\nabla^{m} A\right|\left|\nabla^{i} A\right|\left|\nabla^{j} A\right|\left|\nabla^{k} A\right|$.

In the case $M_{t}=$ graph $w_{t}$, the parabolic system (1) is up to tangential diffeomorphisms equivalent to the quasilinear scalar equation

$$
\frac{d}{d t} w(y, t)=\sqrt{1+|D w|^{2}} \operatorname{div}_{y}\left(\frac{D w}{\sqrt{1+|D w|^{2}}}\right)(y, t) .
$$

Indeed, suppose that $w(y, t)$ is a smooth solution of Eq. (2) on $\Omega \times[0, T)$, where $\Omega$ is some open subset of $R^{n}$. Then we define in the obvious way

$$
\tilde{\mathbf{F}}(y, t)=(y, w(y, t)) \in R^{n+1}, \quad y \in \Omega, t \in[0, T)
$$

and the normal component of $\frac{\partial \tilde{\mathbf{F}}}{\partial t}$ will yield the flow in (1). To be precise let

$$
\varphi: \Omega \times[0, T) \rightarrow R^{n}
$$


be the solution of the following system of ordinary differential equations:

$$
\begin{aligned}
\frac{\partial}{\partial t} \varphi(y, t) & =-\left(\frac{\partial \tilde{\mathbf{F}}}{\partial y}(\varphi(y, t), t)\right)^{-1} \cdot\left(\frac{\partial \tilde{\mathbf{F}}}{\partial t}(\varphi(y, t), t)\right)^{T} \\
\varphi(y, 0) & =y
\end{aligned}
$$

where " $T$ " denotes the tangential component of a vector. Now observe that $v=\sqrt{1+|D w|^{2}}$, and the right hand side of (2) equals $-v H$. We then define

$$
\mathbf{F}(y, t)=\tilde{\mathbf{F}}(\varphi(y, t), t)
$$

and obtain from (2) and (3) that

$$
\begin{aligned}
\frac{d}{d t} \mathbf{F}(y, t) & =\frac{\partial}{\partial t} \tilde{\mathbf{F}}(\varphi(y, t), t)+\frac{\partial \tilde{\mathbf{F}}}{\partial y}(\varphi(y, t), t) \circ \frac{d}{d t} \varphi(y, t) \\
& =\left[\frac{\partial}{\partial t} \tilde{\mathbf{F}}(\varphi(y, t), t)\right] \\
& =\frac{d}{d t} w \cdot v^{-1} \cdot v \\
& =-H \cdot v=\mathbf{H}(y, t) .
\end{aligned}
$$

Notice that the solution of (3) exists and is a diffeomorphism as long as $\varphi(y, t)$ remains in $\Omega$ since $\tilde{\mathbf{F}}$ is a smooth diffeomorphism. The quantity $\left(\frac{d}{d t}\right) \varphi=-H v$ $+H v^{-1} e_{n+1}$ is the projection of $\left(-\left(\frac{d}{d t}\right) \tilde{\mathbf{F}}\right)^{T}$ onto the hyperplane $R^{n}$.

Conversely, given a smooth solution $\mathbf{F}_{t}$ of the mean curvature flow equation which can be written as a graph locally, it is easy to compute that any function $w(y, t)$ with

satisfies

$$
w\left(\mathbf{F}(p, t)-\left\langle\mathbf{F}(p, t), \mathbf{e}_{n+1}\right\rangle \mathbf{e}_{n+1}, t\right)=\left\langle\mathbf{F}(p, t), \mathbf{e}_{n+1}\right\rangle
$$

$$
\begin{aligned}
-H v^{-1} & =\frac{d}{d t} w\left(\mathbf{F}(p, t)-\left\langle\mathbf{F}(p, t), \mathbf{e}_{n+1}\right\rangle \mathbf{e}_{n+1}, t\right) \\
& =\frac{\partial}{\partial t} w\left(\mathbf{F}(p, t)-\left\langle\mathbf{F}(p, t), \mathbf{e}_{n+1}\right\rangle \mathbf{e}_{n+1}, t\right)+H v^{-1}|D w|^{2} .
\end{aligned}
$$

Therefore

$$
\frac{d}{d t} w(y, t)=-H v
$$

which is Eq. (2).

\section{Local gradient estimates}

Let us assume that $M_{0}$ is locally given as a graph over some hyperplane determined by $\omega \in R^{n+1},|\omega|=1$. Then we have the following result. 
2.1 Theorem. Let $R>0$ and $\mathbf{x}_{0} \in R^{n+1}$ be arbitrary and define $\varphi(\mathbf{x}, t)=R^{2}-$ $\left|\mathbf{x}-\mathbf{x}_{0}\right|^{2}-2 n t$. If $\varphi_{+}$denotes the positive part of $\varphi$ we have the estimate

$$
v(\mathbf{x}, t) \varphi_{+}(\mathbf{x}, t) \leqq \sup _{M_{0}} v \varphi_{+}
$$

as long as $v(\mathbf{x}, t)$ is defined everywhere on the support of $\varphi_{+}$.

2.2 Remark. By letting $R \rightarrow \infty$ we obtain the global gradient estimate for entire graphs which was derived in [4] by use of a monotonicity formula, see also [13].

Proof of Theorem 2.1. We may assume without loss of generality that $\mathbf{x}_{0}=0$. For $R>0$ we define $\eta(r)=\left(R^{2}-r\right)^{2}$. Note that $\eta$ satisfies

$$
\eta^{-1}\left(\eta^{\prime}\right)^{2}=4 \text { and } \eta^{\prime \prime}=2
$$

where' denotes differentiation with respect to $r$. If $r=|\mathbf{x}|^{2}+2 n t$ we derive from Lemma 1.1 (i) that

$$
\left(\frac{d}{d t}-\Delta\right) \eta=-\left.\left.2|\nabla| \mathbf{x}\right|^{2}\right|^{2}
$$

Combining (5) with Lemma 1.1 (iii) we obtain

$$
\left(\frac{d}{d t}-\Delta\right) v^{2} \eta=-2|A|^{2} v^{2} \eta-6|\nabla v|^{2} \eta-\left.\left.2|\nabla| \mathbf{x}\right|^{2}\right|^{2} v^{2}-2 \nabla v^{2} \cdot \nabla \eta
$$

Observe that

$$
-2 \nabla v^{2} \cdot \nabla \eta=-6 v \nabla v \nabla \eta+\eta^{-1} \nabla \eta \nabla\left(v^{2} \eta\right)-\eta^{-1}|\nabla \eta|^{2} v^{2}
$$

as well as by (4)

$$
\eta^{-1}|\nabla \eta|^{2} v^{2}=\left.\left.\eta^{-1}\left(\eta^{\prime}\right)^{2}|\nabla| \mathbf{x}\right|^{2}\right|^{2} v^{2}=\left.\left.4|\nabla| \mathbf{x}\right|^{2}\right|^{2} v^{2}
$$

Substituting these identities into (6) implies the estimate

$$
\left(\frac{d}{d t}-\Delta\right) v^{2} \eta \leqq-6|\nabla v|^{2} \eta-\left.\left.6|\nabla| \mathbf{x}\right|^{2}\right|^{2} v^{2}-6 v \nabla v \cdot \nabla \eta+\eta^{-1} \nabla \eta \cdot \nabla\left(v^{2} \eta\right) .
$$

Young's inequality and (4) yield

$$
\begin{aligned}
6 v \nabla v \cdot \nabla \eta & \leqq 6|\nabla v|^{2} \eta+\left.\left.\frac{3}{2} v^{2} \eta^{-1}\left(\eta^{\prime}\right)^{2}|\nabla| \mathbf{x}\right|^{2}\right|^{2} \\
& =6|\nabla v|^{2} \eta+\left.\left.6|\nabla| \mathbf{x}\right|^{2}\right|^{2} v^{2} .
\end{aligned}
$$

If we replace $\eta$ by $\varphi_{+}^{2}$ this computation remains valid on the support of $\varphi_{+}$ as long as $v$ is defined. The weak parabolic maximum principle then implies the result.

Next we shall establish a bound for the gradient in terms of the height over a fixed set in our reference hyperplane. As in [4] we define the height 
of $M_{t}$ with respect to the hyperplane orthogonal to $\omega$ by $u=\langle\mathbf{x}, \omega\rangle$, see Lemma 1.1 (ii).

In contrast, we define the height function $w(y, t)$ to be the height at time $t$ over a fixed point $y$ in this hyperplane.

2.3 Theorem. The gradient of the height function $w$ satisfies the estimate

$$
\begin{aligned}
\sqrt{1+\left|D w\left(y_{0}, t\right)\right|^{2}} \leqq & C_{1}(n) \sup _{B_{R}\left(y_{0}\right)} \sqrt{1+\left|D w_{0}\right|^{2}} \\
& \left.\cdot \exp \left[C_{2}(n) R^{-2} \sup _{[0, T\} B_{R}\left(y_{0}\right) \times\left[0, T^{\prime}\right]} w-w\left(y_{0}, t\right)\right)^{2}\right]
\end{aligned}
$$

where $0 \leqq t \leqq T, B_{R}\left(y_{0}\right)$ is a ball in the hyperplane orthogonal to $\omega$ and $w_{0}$ denotes the initial height function over this hyperplane.

2.4 Remark. This estimate should be compared with the corresponding interior gradient estimate for the equation

$$
\frac{d}{d t} w=\operatorname{div}\left(\frac{D w}{\sqrt{1+|D w|^{2}}}\right)
$$

on $R^{n}$ obtained in [3].

Proof of Theorem 2.3. We are going to adapt the techniques employed in [14] to the parabolic setting and will work with the functions $v$ and $u$ rather than the height function $w$.

Let us first assume that $u<0$ in the set $\bar{M}_{t}=\left\{x \in M_{t} /|\mathbf{x}|^{2}-u^{2} \leqq 1\right\}$ for $t \in[0, T]$ and let $\eta=\eta(x, t) \geqq 0$ be a smooth function which for each $t \in[0, T]$ vanishes outside some compact subset of $R^{n+1}$. Suppose then that max $v \eta$ reaches some value larger than $\max v \eta$ for the first time at $0<t_{0} \leqq T$. At a maximum point on $M_{t_{0}}$ we have

$$
\begin{aligned}
\nabla(v \eta) & =0 \\
\left(\frac{d}{d t}-\Delta\right)(v \eta) & \geqq 0 .
\end{aligned}
$$

In view of the evolution equation for $v$ in Lemma 1.1 (iii) we obtain from (7) and (8) the inequality

$$
0 \leqq v\left(\frac{d}{d t}-\Delta\right) \eta-|A|^{2} v \eta
$$

where we used the relation

$$
\begin{aligned}
-2 \nabla \eta \cdot \nabla v & =-2 v^{-1} \nabla v \cdot \nabla(v \eta)+2 v^{-1}|\nabla v|^{2} \eta \\
& =2 v^{-1}|\nabla v|^{2} \eta
\end{aligned}
$$


Since $v$ and $\eta$ are nonnegative we therefore conclude

$$
\left(\frac{d}{d t}-\Delta\right) \eta \geqq 0
$$

at a maximum point of $v \eta$ on $M_{t_{0}}$.

Let now $\eta=-1+\exp \lambda \varphi$ where the function $\varphi$ will be chosen later and where $\lambda>0$. From (9) we infer

$$
\left(\frac{d}{d t}-\Delta\right) \varphi \geqq \lambda|\nabla \varphi|^{2} .
$$

As in [14] set

$$
\varphi=\begin{array}{ll}
\left(\frac{1}{2 \beta} u+1-\left(|\mathbf{x}|^{2}-u^{2}\right)\right)_{+}, & \mathbf{x} \in \bar{M}_{t}, \beta>0 \text { to be chosen } \\
0, & \text { else. }
\end{array}
$$

Since $u<0$ in $\bar{M}_{t}, \varphi$ is a Lipschitz function vanishing outside $\bar{M}_{t}$. On the set where $\varphi$ is positive we compute

$$
\begin{aligned}
\nabla \varphi & =\frac{1}{2 \beta} \nabla u-\nabla\left(|\mathbf{x}|^{2}-u^{2}\right) \\
|\nabla \varphi|^{2} & =\frac{1}{4 \beta^{2}}|\nabla u|^{2}+\left|\nabla\left(|\mathbf{x}|^{2}-u^{2}\right)\right|^{2}-\frac{1}{\beta} \nabla u \cdot \nabla\left(|\mathbf{x}|^{2}-u^{2}\right) \\
\left(\frac{d}{d t}-\Delta\right) \varphi & =\frac{1}{2 \beta}\left(\frac{d}{d t}-\Delta\right) u-\left(\frac{d}{d t}-\Delta\right)\left(|\mathbf{x}|^{2}-u^{2}\right) .
\end{aligned}
$$

From Lemma 1.1 (i) and (ii) we obtain finally

$$
\left(\frac{d}{d t}-\Delta\right) \varphi=2\left(n-|\nabla u|^{2}\right) \leqq 2 n
$$

Substituting (11) and (12) into (10) we arrive at the inequality

$$
\lambda\left(\frac{1}{4 \beta^{2}}|\nabla u|^{2}-\frac{1}{\beta} \nabla u \cdot \nabla\left(|\mathbf{x}|^{2}-u^{2}\right)\right) \leqq 2 n .
$$

Now note that $\nabla u=\omega-\langle v, \omega\rangle$ and $\nabla\left(|\mathbf{x}|^{2}-u^{2}\right)=2(\mathbf{x}-\langle\mathbf{x}, v\rangle v-u \nabla u)$ such that

$$
\nabla u \cdot \nabla\left(|\mathbf{x}|^{2}-u^{2}\right)=2\left(u-\langle\mathbf{x}, v\rangle\langle v, \omega\rangle-u|\nabla u|^{2}\right) .
$$

Using also that $|\nabla u|^{2}=1-v^{-2}$ we therefore derive

$$
\begin{aligned}
\nabla u \cdot \nabla\left(|\mathbf{x}|^{2}-u^{2}\right) & =2 v^{-1}\left(u v^{-1}-\langle\mathbf{x}, v\rangle\right)=2 v^{-1}(u\langle v, \omega\rangle-\langle\mathbf{x}, v\rangle) \\
& =-2 v^{-1}\langle v, \mathbf{x}-u \omega\rangle \leqq 2 v^{-1}|v||\mathbf{x}-u \omega| \leqq 2 v^{-1}
\end{aligned}
$$


since $|\mathbf{x}-u \omega|^{2}=|\mathbf{x}|^{2}-u^{2} \leqq 1$ in the set under consideration. Thus, again using $|\nabla u|^{2}=1-v^{-2}$ we deduce from (13) that

$$
\lambda\left(\frac{1}{4 \beta^{2}}\left(1-v^{-2}\right)-\frac{2}{\beta} v^{-1}\right) \leqq 2 n
$$

Let now $\lambda=64 n \beta^{2}$. Then at the maximum point of $v \eta$ on $M_{t_{0}}$ we have

Hence

$$
v \leqq 4+16 \beta \text {. }
$$

$$
\begin{aligned}
\max _{\bar{M}_{\iota_{0}}} v \eta & \leqq(4+16 \beta) \sup _{\bar{M}_{\iota_{0}}} \eta \\
& \leqq(4+16 \beta) e^{\lambda}=(4+16 \beta) e^{64 n \beta^{2}}
\end{aligned}
$$

where we used the fact that $\varphi \leqq 1$. This proves that for all $t \in[0, T]$ we have inside $\vec{M}_{t}$ the estimate

$$
v \eta \leqq \sup _{\bar{M}_{0}} v \eta+(4+16 \beta) e^{64 n \beta^{2}}
$$

As described in Sect. 1 this estimate implies an equivalent bound for the height function $w$. At the point $y=0$ in the hyperplane orthogonal to $\omega$ we obtain in equivalence to (15) for $t \in[0, T]$ and arbitrary $\beta>0$

$$
\begin{gathered}
{\left[e^{64 n \beta^{2}\left((2 \beta)^{-1} w(0, t)+1\right)+}-1\right] \sqrt{1+|D w(0, t)|^{2}}} \\
\leqq e^{64 n \beta^{2}}\left(\sup _{B_{1}(0)} \sqrt{1+\left|D w_{0}\right|^{2}}+4+16 \beta\right) .
\end{gathered}
$$

Now choose $\beta=\sup _{t \in[0, T]}-w(0, t)$. We then infer from $(16)$

$$
\sqrt{1+|D w(0, t)|^{2}} \leqq C_{1}(n) \sup _{B_{1}(0)} \sqrt{1+\left|D w_{0}\right|^{2}} \exp \left[C_{2}(n) \sup _{[0, T]}(-w(0, t))^{2}\right]
$$

To achieve the condition $w<0$ on $B_{1}(0)$ we replace $w$ by $w-\sup _{B_{1}(0) \times[0, T]} w(y, t)$ and the estimate on $B_{R}\left(y_{0}\right)$ is then obtained by scaling and translating.

\section{Local curvature and higher order estimates}

In this section we prove that as long as $M_{t}$ can be written as a graph with bounded gradient, the curvature and all its derivatives remain bounded as well.

Let $r=r(\mathbf{x}, t)$ be a smooth nonnegative function such that

$$
\left|\left(\frac{d}{d t}-\Delta\right) r\right| \leqq C(n) \text { and }|\nabla r|^{2} \leqq C(n) r
$$

holds on any surface $M_{t}$ moving by its mean curvature. We begin with a curvature estimate. 
3.1 Theorem. Let $R>0$ be such that $\left\{\mathbf{x} \in M_{t} / r(\mathbf{x}, t) \leqq R^{2}\right\}$ is compact and can be written as a graph over some hyperplane for $t \in[0, T]$. Then for any $t \in[0, T]$ and $0 \leqq \theta<1$ we have the estimate

$$
\sup _{\left\{\left(\mathbf{x} \in M_{t} t r(\mathbf{x}, t) \leqq \theta R^{2}\right)\right.}|A|^{2} \leqq c(n)(1-\theta)^{-2}\left(t^{-1}+R^{-2}\right) \sup _{\left\{\mathbf{x} \in M_{s} / r(\mathbf{x}, s) \leqq R^{2}, s \in[0, t]\right\}} v^{4} .
$$

3.2 Corollary. (i) Let $R>0$ and $0 \leqq \theta<1$ be as above. Then for $\mathbf{x}_{0} \in R^{n+1}$ and $t \in[0, T]$ we have the estimate

$$
\sup _{K\left(\mathbf{x}_{0}, t, \theta R^{2}\right)}|A|^{2} \leqq c(n)(1-\theta)^{-2} t^{-1} \sup _{0 \leqq s \leqq t} \sup _{K\left(\mathbf{x}_{0}, s, R^{2}\right)} v^{4}
$$

where $K\left(\mathbf{x}_{0}, t, \theta R^{2}\right)=\left\{x \in M_{t} /\left|\mathbf{x}-\mathbf{x}_{0}\right|^{2}+2 n t \leqq \theta R^{2}\right\}$.

(ii) Let $R>0$ and $0 \leqq \theta<1$ be as above. Then we have for $t \in[0, T]$ the estimate

$$
\sup _{B_{\theta R}\left(y_{0}\right)}|A|^{2}(t) \leqq c(n)\left(1-\theta^{2}\right)^{-2}\left(\frac{1}{R^{2}}+\frac{1}{t}\right) \sup _{B_{R}\left(y_{0}\right) \times[0, t]} v^{4}
$$

where $B_{R}\left(y_{0}\right)$ denotes a ball in the hyperplane orthogonal to $\omega$.

Proof of Corollary 3.2. We may assume w.l.o.g. that $\mathbf{x}_{0}=0, y_{0}=0$. To prove (i) we note that $r=|\mathbf{x}|^{2}+2 n t$ satisfies condition (17) in view of Lemma 1.1 (i) and the inequality $\left.\left.|\nabla| \mathbf{x}\right|^{2}\right|^{2} \leqq 4|\mathbf{x}|^{2}$. The estimate then follows in view of the fact $t \leqq \frac{R^{2}}{2 n}$ when $r(\mathbf{x}, t) \leqq R^{2}$. To prove the second estimate observe that $r(\mathbf{x}, t)$ $=|\mathbf{x}|^{2}-u^{2}$ satisfies condition (17) in view of Lemma 1.1 (i) and (ii), the inequality $|\nabla u| \leqq 1$ and the fact that $\left|\nabla\left(|\mathbf{x}|^{2}-u^{2}\right)\right|^{2} \leqq 4\left(|\mathbf{x}|^{2}-u^{2}\right)$.

3.3 Remark. (i) By combining Theorem 2.1 with Corollary 3.2 (i) and letting $R \rightarrow \infty$ we obtain the global curvature estimate derived in [4].

(ii) By combining Theorem 2.3 with Corollary 3.2 we can estimate the curvature in terms of a height bound and the initial gradient.

(iii) Corollary 3.2 (ii) includes the estimate in [2] for $H \equiv 0$ as a special case, see also Remark 3.6 (ii).

Proof of Theorem 3.1. From Lemma $1.1(\mathrm{v})$ and Schwarz' inequality we infer that

$$
\left(\frac{d}{d t}-\Delta\right)|A|^{2} \leqq 2|A|^{4}-\left.2|\nabla| A\right|^{2}
$$

Utilizing an idea of Caffarelli, Nirenberg and Spruck in [2] we combine (18) with the evolution equation for the gradient in Lemma 1.1 (iii) to deduce that

$$
\begin{aligned}
& \left(\frac{d}{d t}-\Delta\right)|A|^{2} \varphi\left(v^{2}\right) \\
& \quad \leqq 2\left(\varphi-\varphi^{\prime} v^{2}\right)|A|^{4}-2|\nabla| A \|^{2} \varphi-\left(6 \varphi^{\prime}+4 \varphi^{\prime \prime} v^{2}\right)|A|^{2}|\nabla v|^{2}-2 \nabla|A|^{2} \cdot \nabla \varphi,
\end{aligned}
$$


where $\varphi=\varphi\left(v^{2}\right)$ is a smooth nonnegative function of $v^{2}$ to be determined later and 'denotes derivatives with respect to $v^{2}$. The last term can be estimated by

$$
\begin{aligned}
-2 \nabla|A|^{2} \cdot \nabla \varphi= & -\varphi^{-1} \nabla \varphi \cdot \nabla\left(|A|^{2} \varphi\right)+\varphi^{-1}|\nabla \varphi|^{2}|A|^{2}-4|A| v \varphi^{\prime} \nabla|A| \cdot \nabla v \\
\leqq & -\varphi^{-1} \nabla \varphi \cdot \nabla\left(|A|^{2} \varphi\right)+4 \varphi^{-1}\left(\varphi^{\prime}\right)^{2} v^{2}|\nabla v|^{2}|A|^{2}+\left.2|\nabla| A\right|^{2} \varphi \\
& +2|A|^{2} v^{2} \varphi^{-1}\left(\varphi^{\prime}\right)^{2}|\nabla v|^{2} \\
= & -\varphi^{-1} \nabla \varphi \cdot \nabla\left(|A|^{2} \varphi\right)+\left.2|\nabla| A\right|^{2} \varphi+6 \varphi^{-1}\left(\varphi^{\prime}\right)^{2} v^{2}|A|^{2}|\nabla v|^{2}
\end{aligned}
$$

Hence we obtain

$$
\begin{aligned}
& \left(\frac{d}{d t}-\Delta\right)|A|^{2} \varphi \leqq 2\left(\varphi-\varphi^{\prime} v^{2}\right)|A|^{4} \\
& \quad-\varphi^{-1} \nabla \varphi \cdot \nabla\left(|A|^{2} \varphi\right)-\left(6 \varphi^{\prime}\left(1-\varphi^{-1} \varphi^{\prime} v^{2}\right)+4 \varphi^{\prime \prime} v^{2}\right)|A|^{2}|\nabla v|^{2}
\end{aligned}
$$

As in [2] we let $\varphi\left(v^{2}\right)=\frac{v^{2}}{1-k v^{2}}, k>0$ to be chosen. Observe that then

$$
\begin{aligned}
\varphi-\varphi^{\prime} v^{2} & =-k \varphi^{2}, \\
6 \varphi^{\prime}\left(1-\varphi^{-1} \varphi^{\prime} v^{2}\right)+4 \varphi^{\prime \prime} v^{2} & =\frac{2 k}{\left(1-k v^{2}\right)^{2}} \varphi,
\end{aligned}
$$

and

$$
\varphi^{-1} \nabla \varphi=2 \varphi v^{-3} \nabla v
$$

Substituting these identities into (19) we derive for $g=|A|^{2} \varphi\left(v^{2}\right)$ the inequality

$$
\left(\frac{d}{d t}-\Delta\right) g \leqq-2 k g^{2}-\frac{2 k}{\left(1-k v^{2}\right)^{2}}|\nabla v|^{2} g-2 \varphi v^{-3} \nabla v \cdot \nabla g .
$$

Now let $\eta=\eta(r)=\left(R^{2}-r\right)^{2}$ where $r=r(\mathbf{x}, t)$ satisfies (17). Since by (4) and (17)

$$
\begin{aligned}
\left(\frac{d}{d t}-\Delta\right) \eta & =\eta^{\prime}\left(\frac{d}{d t}-\Delta\right) r-\eta^{\prime \prime}|\nabla r|^{2} \\
& \leqq 2 c(n) R^{2}-2|\nabla r|^{2}
\end{aligned}
$$

we compute from (20)

$$
\begin{aligned}
\left(\frac{d}{d t}-\Delta\right) g \eta \leqq & -2 k g^{2} \eta-\frac{2 k}{\left(1-k v^{2}\right)^{2}}|\nabla v|^{2} g \eta-2 \varphi v^{-3} \nabla v \cdot \nabla g \cdot \eta \\
& +c(n) R^{2} g-2 g|\nabla r|^{2}-2 \nabla g \cdot \nabla \eta
\end{aligned}
$$

where $c(n)$ denotes constants depending on $n$ only. The last term can be written as

$$
-2 \nabla g \cdot \nabla \eta=-2 \eta^{-1} \nabla \eta \cdot \nabla(g \eta)+8 g|\nabla r|^{2}
$$


in view of (4). We also estimate

$$
\text { (23) } \begin{aligned}
- & 2 \varphi v^{-3} \nabla v \cdot \nabla g \eta \\
& =-2 \varphi v^{-3} \nabla v \cdot \nabla(g \eta)+2 \varphi v^{-3} g \eta^{\prime} \nabla v \cdot \nabla r \\
& \leqq-2 \varphi v^{-3} \nabla v \cdot \nabla(g \eta)+\frac{2 k}{\left(1-k v^{2}\right)^{2}}|\nabla v|^{2} g \eta+2 \varphi^{2} v^{-6} \frac{\left(1-k v^{2}\right)^{2}}{k} g|\nabla r|^{2} \\
& =-2 \varphi v^{-3} \nabla v \cdot \nabla(g \eta)+\frac{2 k}{\left(1-k v^{2}\right)^{2}}|\nabla v|^{2} g \eta+\frac{2}{k v^{2}} g|\nabla r|^{2}
\end{aligned}
$$

where we again used (4) and the fact that

$$
\varphi^{2} v^{-6}\left(1-k v^{2}\right)^{2}=v^{-2}
$$

Substituting (22) and (23) into (21) and using (17) we derive

$$
\left(\frac{d}{d t}-\Delta\right) g \eta \leqq-2 k g^{2} \eta-2\left(\varphi v^{-3} \nabla v+\eta^{-1} \nabla \eta\right) \cdot \nabla(g \eta)+c(n)\left(\left(1+\frac{1}{k v^{2}}\right) r+R^{2}\right) g
$$

and hence

$$
\begin{aligned}
\left(\frac{d}{d t}-\Delta\right) \operatorname{tg} \eta \leqq & -2 k g^{2} \eta t-2\left(\varphi v^{-3} \nabla v+\eta^{-1} \nabla \eta\right) \cdot \nabla(g \eta t) \\
& +c(n)\left(\left(1+\frac{1}{k v^{2}}\right) r+R^{2}\right) t g+g \eta
\end{aligned}
$$

At a point where $m(T)=\sup _{0 \leqq t \leqq T} \sup _{\left.\left\{\mathbf{x} \in M_{t}\right\} r(\mathbf{x}, t) \leqq R^{2}\right\}} \operatorname{tg} \eta$ is attained for $t_{0}>0$ we compute

$$
2 k g^{2} \eta t_{0} \leqq c(n)\left(1+\frac{1}{k v^{2}}\right) R^{2} g t_{0}+g \eta
$$

Multiplying by $\eta \frac{t_{0}}{2 k}$ yields since $t_{0} \leqq T$

$$
m(T)^{2} \leqq \frac{c(n)}{2 k}\left(\left(1+\frac{1}{k v^{2}}\right) R^{2} T+\eta\right) m(T)
$$

Since $\eta \leqq R^{4}$ we arrive at

$$
g \eta T \leqq \frac{c(n)}{2 k}\left(\left(1+\frac{1}{k v^{2}}\right) R^{2} T+R^{4}\right)
$$

in the set $\left\{\mathrm{x} \in M_{T} / r(\mathbf{x}, T) \leqq R^{2}\right\}$. Let now

$$
k=\frac{1}{2} \inf _{\left\{\mathbf{x} \in M_{t} / r(\mathbf{x}, t) \leqq R^{2}, t \in[0, T]\right\}} v^{-2} .
$$


Since $\varphi\left(v^{2}\right) \geqq 1$ and $\eta \geqq(1-\theta)^{2} R^{4}$ in $\left\{\mathbf{x} \in M_{T} / r(\mathbf{x}, T) \leqq \theta R^{2}\right\}$ we finally obtain from (24) replacing $T$ by any $t \in[0, T]$

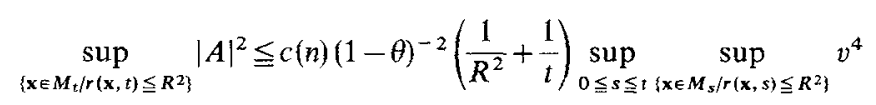

as desired.

We will now extend these estimates to all derivatives of $A$.

3.4 Theorem. Let $r=r(\mathbf{x}, t) \geqq 0$ be as in (17) and let $R>0$ be such that $\left\{\mathbf{x} \in M_{t} / r(\mathbf{x}, t) \leqq R^{2}\right\}$ is compact and can be written as a graph over some hyperplane for $t \in[0, T]$. Then for any $m \geqq 0,0 \leqq \theta<1$ and $t \in[0, T]$ we have the estimate

$$
\begin{aligned}
& \sup _{\left\{\mathbf{x} \in M_{\mathrm{t}} / r(\mathbf{x}, t) \leqq R^{2}\right\}}\left|\nabla^{m} A\right|^{2} \leqq c_{m}\left(\frac{1}{R^{2}}+\frac{1}{t}\right)^{m+1} \\
& \text { where } c_{m}=c_{m}\left(\theta, n, m, \sup _{\left\{\mathbf{x} \in M_{s} / r(\mathbf{x}, s) \leqq R^{2}, s \in[0, t]\right\}} v\right)
\end{aligned}
$$

As in Corollary 2.2 setting $r=\left|\mathbf{x}-\mathbf{x}_{0}\right|^{2}+2 n t$ or $r=|\mathbf{x}|^{2}-u^{2}$ implies

3.5 Corollary. (i) Let $R>0$ and $0 \leqq \theta<1, m>0$ be as above. Then we have for $\mathbf{x}_{0} \in R^{n+1}, t \in[0, T]$ the estimate

$$
\sup _{K\left(\mathbf{x}_{0}, t, \theta \mathbf{R}^{2}\right)} t^{m+1}\left|\nabla^{m} A\right|^{2} \leqq c_{m}
$$

where $K\left(\mathbf{x}_{0}, t, \theta R^{2}\right)=\left\{\mathbf{x} \in M_{\mathrm{t}} /\left|\mathbf{x}-\mathbf{x}_{0}\right|^{2}+2 n t \leqq R^{2}\right\}$ and $c_{m}$ is as in Theorem 3.4.

(ii) Let $R>0,0 \leqq \theta<1$ and $m \geqq 0$ be as above. Then we have for $t \in[0, T]$ the estimate

$$
\sup _{B_{\theta_{\mathbf{R}}\left(y_{0}\right)}}\left|\nabla^{m} A\right|^{2}(t) \leqq c_{m}\left(\frac{1}{R^{2}}+\frac{1}{t}\right)^{m+1}
$$

where $B_{\mathbf{R}}\left(y_{0}\right)$ denotes a ball in the hyperplane orthogonal to $\omega$ and $c_{m}$ is as above.

3.6 Remark. (i) By combining Theorem 2.1 and Corollary 3.5 (i) and letting $R \rightarrow \infty$ we obtain the global estimates derived in [4].

(ii) From the proof of Theorem 3.4 we infer that the constant $c_{m}$ is of the form $c_{m}=c(n, \theta, m) c_{0}^{\alpha(m)}$ where $\alpha(m)$ is a positive integer (bounded by $2^{m}$ ) and $c_{0}$ is s.t.

$$
\sup _{\left\{\mathbf{x} \in M_{s} / r(\mathbf{x}, s) \leqq R^{2}\right\}}|A|^{2} \leqq c_{0}\left(\frac{1}{R^{2}}+\frac{1}{s}\right)
$$

for any $s \in[0, t]$. The graph property of $M_{t}$ is only used to bound $|A|^{2}$ as in Theorem 3.1. One determines an optimal exponent $\alpha(m)$ by estimating the last term in the evolution equation for $\left|\nabla^{m} A\right|^{2}$ (see Lemma 1.1 (vi)) similarly as in Proposition 4.4 of [4].

(iii) Since minimal hypersurfaces $(H \equiv 0)$ are stationary solutions of (1) the estimates 3.5 (i), (ii) for $m \geqq 0$ with $t$ proportional to $R^{2}$ provide an interior regularity theory for solutions of the minimal surface equation obtained entirely from 
geometric computations. To minimize the exponent of $v$ in the $\left|\nabla^{m} A\right|$-estimate one uses the curvature bound in [5] and then finds the optimal exponent $\alpha(m)$ as in (ii) above.

Proof of Theorem 3.4. We proceed by induction on $m$ in a similar way to that in [4]. The case $m=0$ was proved in Theorem 3.1. For fixed $R>0$ let $\psi(t)$ $=\frac{R^{2} t}{\left(R^{2}+t\right)}$. Suppose

$$
\sup _{\left\{\mathbf{x} \in M_{t} / r(\mathbf{x}, t) \leqq R^{2}, t \in[0, T]\right\}} \psi^{k}\left|\nabla^{k-1} A\right|^{2} \leqq c_{k-1}
$$

has been established for $1 \leqq k \leqq m$. We then want to estimate $\psi^{m+1}\left|\nabla^{m} A\right|^{2}$ in $\left\{\mathbf{x} \in M_{t} / r(\mathbf{x}, t) \leqq \theta R^{2}\right\}$. From Lemma 1.1 (vi) we obtain for $m \geqq 1$

$$
\begin{aligned}
& \left(\frac{d}{d t}-\Delta\right) \psi^{m+1}\left|\nabla^{m} A\right|^{2} \leqq-2 \psi^{m+1}\left|\nabla^{m+1} A\right|^{2} \\
& \quad+c(m, n) \psi^{m+1} \sum_{i+j+k=m}\left|\nabla^{i} A\right|\left|\nabla^{j} A\right|\left|\nabla^{k} A\right|\left|\nabla^{m} A\right|+\left(\frac{d}{d t} \psi^{m+1}\right)\left|\nabla^{m} A\right|^{2} .
\end{aligned}
$$

In $\left\{\mathbf{x} \in M_{t} / r(\mathbf{x}, t) \leqq \theta R^{2}\right\}$ we have by (25)

$$
\psi^{m+1} \sum_{i+j+k=m}\left|\nabla^{i} A\right|\left|\nabla^{j} A\right|\left|\nabla^{k} A\right|\left|\nabla^{m} A\right| \leqq c \sum_{k \leqq m} \psi^{k}\left|\nabla^{k} A\right|^{2}
$$

similarly as in [4], where $c=c\left(n, m, c_{0}, \ldots, c_{m-1}\right)$. Since

$$
\frac{d}{d t} \psi^{m+1}=(m+1) \psi^{m} \frac{R^{4}}{\left(R^{2}+t\right)^{2}} \leqq(m+1) \psi^{m}
$$

we infer in $\left\{\mathbf{x} \in M_{t} / r(\mathbf{x}, t) \leqq R^{2}\right\}$ the inequality

$$
\left(\frac{d}{d t}-\Delta\right) \psi^{m+1}\left|\nabla^{m} A\right|^{2} \leqq-2 \psi^{m+1}\left|\nabla^{m+1} A\right|^{2}+C \sum_{k \leqq m} \psi^{k}\left|\nabla^{k} A\right|^{2},
$$

where $c=c\left(n, m, c_{0}, \ldots, c_{m-1}\right)$. Following the work of Shi in [17] we now define

$$
f=\psi^{m+1}\left|\nabla^{m} A\right|^{2}\left(A+\psi^{m}\left|\nabla^{m-1} A\right|^{2}\right),
$$

where $A>0$ will be chosen appropriately. We then compute, using again (25)

$$
\begin{aligned}
\left(\frac{d}{d t}-\Delta\right) f \leqq & -2 \psi^{m+1}\left|\nabla^{m+1} A\right|^{2}\left(A+\psi^{m}\left|\nabla^{m-1} A\right|^{2}\right) \\
& +C \sum_{k \leqq m} \psi^{k}\left|\nabla^{k} A\right|^{2}\left(A+\psi^{m}\left|\nabla^{m-1} A\right|^{2}\right) \\
& -2 \psi^{2 m+1}\left|\nabla^{m} A\right|^{4}+\sum_{k \leqq m-1} \psi^{k}\left|\nabla^{k} A\right|^{2} \psi^{m+1}\left|\nabla^{m} A\right|^{2} \\
& -2 \psi^{2 m+1} \nabla\left|\nabla^{m} A\right|^{2} \cdot \nabla\left|\nabla^{m-1} A\right|^{2}
\end{aligned}
$$


The last term we estimate by

$$
\begin{aligned}
2 \psi^{2 m+1} \nabla\left|\nabla^{m} A\right|^{2} \cdot \nabla\left|\nabla^{m-1} A\right|^{2} \leqq & 2 \psi^{m+1}\left|\nabla^{m} A\right|^{2}\left(A+\psi^{m}\left|\nabla^{m-1} A\right|^{2}\right) \\
& +8 \psi^{2 m+1} \frac{\psi^{m}\left|\nabla^{m-1} A\right|^{2}}{A+\psi^{m}\left|\nabla^{m-1} A\right|^{2}}\left|\nabla^{m} A\right|^{4} .
\end{aligned}
$$

Noting that

$$
\frac{\psi^{m}\left|\nabla^{m-1} A\right|^{2}}{\Lambda+\psi^{m}\left|\nabla^{m-1} A\right|^{2}} \leqq \frac{c_{m-1}}{\Lambda+c_{m-1}}
$$

we obtain in $\left\{\mathbf{x} \in M_{t} / r(\mathbf{x}, t) \leqq R^{2}\right\}$ that

$$
\begin{aligned}
\left(\frac{d}{d t}-\Delta\right) f \leqq & -2 \psi^{-1}\left(1-\frac{4 c_{m-1}}{A+c_{m-1}}\right)\left(\psi^{m+1}\left|\nabla^{m} A\right|^{2}\right)^{2} \\
& +c \psi^{-1}\left(\sum_{k \leqq m} \psi^{k+1}\left|\nabla^{k} A\right|^{2}\left(A+\psi^{m}\left|\nabla^{m-1} A\right|^{2}\right)\right. \\
& \left.+\sum_{k \leqq m-1} \psi^{k+1}\left|\nabla^{k} A\right|^{2} \psi^{m+1}\left|\nabla^{m} A\right|^{2}\right)
\end{aligned}
$$

Setting $A=7 c_{m-1}+1$ and collecting terms we obtain in view of (25) in $\left\{\mathbf{x} \in M_{t} / r(\mathbf{x}, t) \leqq R^{2}\right\}$

$$
\left(\frac{d}{d t}-\Delta\right) f \leqq-\psi^{-1}\left(A+\psi^{m}\left|\nabla^{m-1} A\right|^{2}\right)^{-2} f^{2}+c \psi^{-1}(1+f),
$$

where $c$ depends on $n, m, c_{0}, \ldots, c_{m-1}$. Applying now (25) to the $f^{2}$-term and using Young's inequality on the second term we arrive at

$$
\left(\frac{d}{d t}-\Delta\right) f \leqq-\psi^{-1}\left(\delta f^{2}-c\right)
$$

where $\delta$ and $c$ depend on $n, m, c_{0}, \ldots, c_{m-1}$. Similarly as in the proof of Theorem 3.1 we compute for $\eta=\left(R^{2}-r\right)^{2}$ using (17) that

$$
\left(\frac{d}{d t}-\Delta\right) f \eta \leqq \psi^{-1}\left(-\delta f^{2}+c\right) \eta-2 \eta^{-1} \nabla \eta \cdot \nabla(f \eta)+c(n) R^{2} f
$$

Since $\psi(0)=0$ we have $f \eta \equiv 0$ at $t=0$. At the point where $m(T)$ $=\sup _{0 \leqq t \leqq T} \sup _{\left\{\mathbf{x} \in M_{t} / r(\mathbf{x}, t) \leqq R^{2}\right\}} f \eta$ is attained for $t_{0}>0$ we compute

$$
f^{2} \eta \leqq \frac{1}{\delta}\left(c \eta+c(n) R^{2} f \psi\right)
$$

Multiplying by $\eta$ and using $\eta \leqq R^{4}$ and $\psi \leqq R^{2}$ we obtain since $\delta=\delta\left(n, m_{0}, c_{0}, \ldots, c_{m-1}\right)$ the estimate

$$
f^{2} \eta^{2} \leqq c\left(R^{8}+R^{4} f \eta\right)
$$

where $c=c\left(n, m, c_{0}, \ldots, c_{m-1}\right)$. Hence, by Young's inequality we derive 
and therefore

$$
f \eta \leqq c R^{4}
$$

in $\left\{\mathbf{x} \in M_{T} / r(\mathbf{x}, T) \leqq R^{2}\right\}$ with $c=c\left(n, m, c_{0}, \ldots, c_{m-1}\right)$. Thus we finally arrive at

$$
\sup _{\left\{\mathbf{x} \in M_{T} / \mathbf{r}(\mathbf{x}, T) \leqq R^{2}\right\}} \psi^{m+1}\left|\nabla^{m} A\right|^{2} \leqq c(1-\theta)^{-2}
$$

where $c=c\left(n, m, c_{0}, \ldots, c_{m-1}\right)$ and where we used the fact that $\eta \geqq(1-\theta)^{2} R^{4}$ and $A \geqq 1$. Replacing $T$ by any $t \in[0, T]$ yields the result.

If we assume additional smoothness of $M_{t}$ we can improve the time dependence of the higher derivative estimates. In particular, it is not necessary to assume that $M_{t}$ can be locally written as a graph.

3.7 Theorem. Let $r=r(\mathbf{x}, t) \geqq 0$ be as in (17) and let $R>0$ be such that $\left\{\mathbf{x} \in M_{t} / r(\mathbf{x}, t) \leqq R^{2}\right\}$ is compact for $t \in[0, T]$. Then for $0 \leqq \theta<1, t \in[0, T]$ and any integers $\ell \geqq 0, m \geqq 0$ we have the estimate

$$
\begin{aligned}
& \sup _{\left\{\mathbf{x} \in M_{t} / r(\mathbf{x}, t) \leqq R^{2}\right\}}\left|\nabla^{m+\ell} A\right|^{2} \leqq c\left(1+\frac{1}{R^{2}}+\frac{1}{t}\right)^{\ell} \\
& \text { where } c=c\left(m, \ell, n, \theta, \sup _{\left\{\mathbf{x} \in M_{s}, r(\mathbf{x}, s) \leqq R^{2}, s \in[0, t]\right\}} \sum_{i=0}^{m}\left|\nabla^{i} A\right|^{2}\right) .
\end{aligned}
$$

In particular, we obtain the estimate

$$
\sup _{\boldsymbol{K}\left(\mathbf{x}_{0}, t, \theta R^{2}\right)}\left|\nabla^{m+\ell} A\right|^{2} \leqq c t^{-\ell} .
$$

3.8 Remark. For $m=0$ and $\ell=1$ we have

$$
c=c(n, \theta) . \sup _{\left\{\mathbf{x} \in M_{s} / r(\mathbf{x}, s) \leqq R^{2}, s \in[0, t]\right\}}|A|^{2} .
$$

For general $m$ and $\ell$ the constant can be optimized as outlined in Remark 3.6 (ii).

Proof of Theorem 3.7. Observe that similar as in the proof of Theorem 3.4 the function $f=\psi^{\ell}\left|\nabla^{m+\ell} A\right|^{2}\left(A+\psi^{\ell-1}\left|\nabla^{m+\ell-1} A\right|^{2}\right)$ with $\psi=\left(1+\frac{1}{R^{2}}+\frac{1}{t}\right)^{-1}$ satisfies an inequality of the form

$$
\left(\frac{d}{d t}-\Delta\right) f \leqq \psi^{-1}\left(\delta f^{2}-C\right)
$$

\section{Flow of complete surfaces}

In this section we want to establish a number of results for complete, noncompact surfaces moving by mean curvature. In particular, we will use the interior estimates from the previous section to obtain a short time existence result for arbitrary immersed, complete initial surfaces assuming only a uniform local Lipschitz condition. A similar approach to a short time existence result is also 
known to R. Hamilton [9]. Furthermore, we will derive a general maximum principle for heat equations on Riemannian manifolds with a time dependent metric.

We begin with a result for the Dirichlet problem. Let $M^{n}$ be a smooth manifold with boundary $\partial M^{n}$ a compact $(n-1)$ dimensional smooth submanifold such that $\bar{M}^{n}=M^{n} \cup \partial M^{n}$ is compact. Then the following shorttime existence result holds.

4.1 Proposition. Suppose that $\mathbf{F}_{0}: \bar{M}^{n} \rightarrow R^{n+1}$ is a smooth isometric immersion. Then there is $T_{0}>0$ such that the initial value problem

$$
\begin{array}{ll}
\frac{d}{d t} \mathbf{F}(p t)=\mathbf{H}(p, t), & p \in M^{n}, \quad 0<t<T_{0} \\
\mathbf{F}(p, 0)=\mathbf{F}_{0}(p), & p \in M^{n} \\
\mathbf{F}(p, t)=\mathbf{F}_{0}(p), & p \in \partial M^{n}, 0 \leqq t \leqq T_{0}
\end{array}
$$

has a unique smooth solution on $0 \leqq t<T_{0}$. The constant $T_{0}>0$ only depends on a bound for the second fundamental form of $M_{0}$ and $\partial M_{0}$.

Proof. Since $\mathbf{F}_{0}$ is smooth there is a tubular neighbourhood of $M_{0}$ with Gaussian coordinates $\hat{x}(p)=\left(x_{1}, \ldots, x_{n}\right), y$ such that at a point $q$ in this neighbourhood $y(q)$ is the length of the geodesic arc perpendicular to $M_{0}$ through $q$ and $\hat{x}(q)$ denotes the coordinate vector at the base point of the geodesic on $M_{0}$. Let $\omega(\hat{x})$ be the unit normal to $M_{0}$ at $\hat{x}$. In these coordinates consider the scalar initial value problem

$$
\begin{array}{ll}
\frac{d}{d t} w(\hat{x}, t)=\langle v(\hat{x}, t), \omega(\hat{x})\rangle^{-1} H(\hat{x}, t), & \hat{x} \in M_{0}, \quad 0<t<T_{0} \\
w(\hat{x}, 0)=0, & \hat{x} \in M_{0} \\
w(\hat{x}, t)=0, & \hat{x} \in \partial M_{0}, 0 \leqq t<T_{0}
\end{array}
$$

where $v$ and $H$ are the unit normal and the mean curvature respectively of the hypersurface given by $w$. This is a quasilinear second order equation which is uniformly parabolic as long as $\langle v, \omega\rangle^{-1}$ remains uniformly bounded. At time $t=0$ we have $\langle\nu, \omega\rangle \equiv 1$ and standard arguments yield an a priori estimate for $\langle\nu, \omega\rangle^{-1}$ on a time interval $\left[0, T_{0}\right)$ which only depends on a bound for the curvature of $M_{0}$ and $\partial M_{0}$. Note that an interior estimate for the gradient function $\langle v, \omega\rangle^{-1}$ also follows from Theorem 2.3. Then the results of Ladyzhenskaya et al. [15] apply and yield a smooth solution of (27) on [0, $T_{0}$ ). Using an appropriate tangential diffeomorphism exactly as in Sect. 1 we then construct a smooth solution of the original problem (26).

Now let $\mathbf{F}_{0}: M^{n} \rightarrow R^{n+1}$ be the smooth isometric immersion of a complete hypersurface with bounded curvature in $R^{n+1}$. Fix a point $p_{0} \in M^{n}$ and as in [17] choose a family of domains $\left\{D_{k} \mid k=1,2,3, \ldots\right\}$ on $M^{n}$ such that for each $k$ the boundary $\partial D_{k}$ is a compact $C_{\infty},(n-1)$-dimensional submanifold of $M^{n}$ and

(i) $\bar{D}_{k}=D_{k} \cup \partial D_{k}$ is a compact subset of $M^{n}$,

(ii) the geodesic ball $B(p, k)$ is contained in $D_{k}$.

For each $k=1,2,3, \ldots$ let $\mathbf{F}_{t}^{k}$ be the solution of the Dirichlet problem (26) with initial data $\mathbf{F}_{0}$ on $\bar{D}_{k}$. Proposition 4.1 guarantees that such a solution exists for each $k$ on a time interval $\left[0, T_{0}\right)$ with $0<T_{0}$ independent of $k$. Since $M_{0}$ has bounded curvature our interior estimates in Theorem 3.4 apply and we 
may take the limit $k \rightarrow \infty$ to obtain a smooth solution of the mean curvature flow (1) with initial data $M_{0}$.

Furthermore, in view of the interior gradient estimate in Theorem 2.3 a simple approximation argument shows that it is sufficient for the short time existence result to assume the following uniform local Lipschitz condition on $M_{0}$.

For $q \in M^{n}$ let $U_{r, q}$ be the $q$-component of $\mathbf{F}_{0}^{-1}\left(B_{r}^{n+1}\left(\mathbf{F}_{0}(q)\right)\right)$. We say that $M_{0}$ satisfies a uniform local Lipschitz condition if there are fixed numbers $r_{0}>0$, $C_{0}<\infty$ such that for each $q \in M^{n}, \mathbf{F}_{0}\left(U_{r_{0}, q}\right)$ can be written as the graph of a Lipschitz function over a hyperplane in $R^{n+1}$ through $\mathbf{F}_{0}(q)$ with Lipschitz constant less than $C_{0}$.

Using Theorem 2.3 and the special time dependence of the curvature estimates in Corollary 3.5 (ii) we obtain after approximation:

4.2 Theorem. Let $\mathbf{F}_{0}: M^{n} \rightarrow R^{n+1}$ satisfy a uniform local Lipschitz condition. Then the mean curvature flow problem

$$
\begin{aligned}
& \frac{d}{d t} \mathbf{F}(p, t)=\mathbf{H}(p, t), \quad p \in M^{n}, t>0 \\
& \mathbf{F}(p, 0)=\mathbf{F}_{0}(p)
\end{aligned}
$$

has a solution $\mathbf{F}_{t}$ on some time interval $\left[0, T_{0}\right) . \mathbf{F}_{t}$ is smooth for $t>0$ and at $t=0$ it is Hölder continuous in time with Hölder exponent $\alpha=\frac{1}{2}$.

We will now adapt a method of Liao and Tam [14] to establishing a maximum principle for heat equations which not only applies to our noncompact hypersurfaces, but to general Riemannian manifolds with a time dependent metric. Notice that a maximum principle for the mean curvature flow was obtained in [4] assuming more restrictive growth properties of the solution. Let $M^{n}$ be a complete, non-compact Riemannian manifold with time dependent metric $g(t)=\left\{g_{i j}(t)\right\}$ for $0 \leqq t<T$. We denote by $B_{r}^{t}(p)$ the geodesic ball of radius $r$ centred at $p$ at time $t$ and will often use a superscript or subscript $t$ to denote other time dependent quantities.

4.3 Theorem. Suppose that the manifold $M^{n}$ with Riemannian metrics $g(t)$ satisfies a uniform volume growth restriction, namely

$$
\operatorname{vol}^{t}\left(B_{r}^{t}(p)\right) \leqq \exp \left(k\left(1+r^{2}\right)\right)
$$

holds for some point $p \in M^{n}$ and a uniform constant $k>0$ for all $t \in[0, T]$.

Let be a function on $M^{n} \times[0, T]$ which is smooth on $M^{n} \times(0, T]$ and continuous on $M^{n} \times[0, T]$. Assume that $f$ and $g(t)$ satisfy

(i) $\frac{d}{d t} f \leqq \Delta^{t} f+\mathbf{a} \cdot \nabla f+b f$ where the function $b$ satisfies $\sup _{M^{n \times[0, T]}}|b| \leqq \alpha_{0}$ for some $\alpha_{0}<\infty$ and the vector field a satisfies $\sup _{M^{n \times[0, T]}}|\mathbf{a}| \leqq \alpha_{1}$ for some $\alpha_{1}<\infty$,

(ii) $f(p, 0) \leqq 0$ for all $p \in M^{n}$,

(iii) $\int_{0}^{T}\left(\int_{M} \exp \left(-\alpha_{2}^{2} r^{t}(p, y)^{2}\right)|\nabla f|^{2}(y) d \mu_{t}\right) d t<\infty$, for some $\alpha_{2}>0$

(iv) $\sup _{M^{n \times[0, T]}}\left|\frac{d}{d t} g_{i j}\right| \leqq \alpha_{3}$, for some $\alpha_{3}<\infty$.

Then we have $f \leqq 0$ on $M^{n} \times[0, T]$. 
Proof. First notice that in view of (iv) and ([8, Lemma 14]) all metrics $g(t)$ are uniformly equivalent, i.e. there is a constant $\alpha_{4}$ depending only on $\alpha_{3}$ and $T$ such that for any vector $v(q), q \in M^{n}$ we have for $t \in[0, T]$

$$
\alpha_{4}^{-1} g_{i j}(q, 0) v^{i}(q) v^{j}(q) \leqq g_{i j}(q, t) v^{i}(q) v^{j}(q) \leqq \alpha_{4} g_{i j}(q, 0) v^{i}(q) v^{j}(q) .
$$

We then proceed as in [16] and let $0<\eta<\min \left(T, \frac{1}{64 K}, \frac{1}{32 \alpha_{2}}\right)$ be a fixed constant. Define for $\theta>0$ to be chosen

$$
h(y, s)=-\frac{\theta r_{s}^{2}(p, y)}{4(2 \eta-s)}, \quad 0<s<\eta
$$

where $r_{s}(p, y)$ is the distance between $p$ and $y$ at time $s$. Then $h$ is Lipschitz continuous and

$$
\begin{aligned}
\frac{d}{d s} h & =-\frac{\theta r_{s}^{2}(p, y)}{4(2 \eta-s)^{2}}-\frac{\theta r_{s}}{2(2 \eta-s)} \frac{d}{d s} r_{s} \\
& =-\theta^{-1}|\nabla h|^{2}-\frac{\theta r_{s}}{2(2 \eta-s)} \frac{d}{d s} r_{s} .
\end{aligned}
$$

Now observe that for any curve $\gamma$ with length $l(s)$ we have

$$
\left|\frac{d}{d s} l(s)\right|=\left|\frac{d}{d s} \int_{\gamma} \sqrt{g(\dot{\gamma}, \dot{\gamma})} d \tau\right| \leqq \frac{1}{2} \alpha_{3} l(s)
$$

such that in particular

$$
\left|\frac{d}{d s} r_{s}\right| \leqq \frac{1}{2} \alpha_{3} r_{s}
$$

We therefore conclude that

$$
\frac{d}{d s} h \leqq-\theta^{-1}|\nabla h|^{2}+\theta^{-1} \alpha_{3}|\nabla h|^{2} \cdot(2 \eta-s) .
$$

Using then $\eta \leqq \frac{1}{2 \alpha_{3}}$ we obtain for $\theta=\frac{1}{4}$

$$
\frac{d}{d s} h \leqq-2|\nabla h|^{2}
$$

Proceeding similarly as in [16] we obtain for $f_{K}=\max \{\min (f, K), 0\}$ and some smooth time independent compactly supported function $\varphi$ on $M^{n}$ that for $0<\varepsilon$ $<\eta$

$$
\begin{aligned}
& \int_{\varepsilon}^{\eta} e^{-\beta s}\left(\int_{M} \varphi^{2} e^{h} f_{K}\left(\Delta f-\frac{\partial f}{\partial s}\right) d \mu_{s}\right) d s \\
& \quad \geqq-\alpha_{1} \int_{\varepsilon}^{\eta} e^{-\beta s}\left(\int_{M} \varphi^{2} e^{h} f_{K}|\nabla f| d \mu_{s}\right) d s-\alpha_{0} \int_{\varepsilon}^{\eta} e^{-\beta s}\left(\int_{M} \varphi^{2} e^{h} f_{K} f d \mu_{s}\right) d s
\end{aligned}
$$


where $\beta>0$ will be chosen later and where we used that $f_{K} f \geqq 0$. Performing an integration by parts yields

$$
\begin{aligned}
0 \leqq & -\int_{\varepsilon}^{\eta} e^{-\beta s}\left(\int_{M} \varphi^{2} e^{h} f_{K}\left\langle\nabla f_{K}, \nabla f\right\rangle d \mu_{s}\right) d s \\
& -\int_{\varepsilon}^{\eta} e^{-\beta s}\left(\int_{M} \varphi^{2} e^{h} f_{K}\langle\nabla h, \nabla f\rangle d \mu_{s}\right) d s \\
& -2 \int_{\varepsilon}^{\eta} e^{-\beta s}\left(\int_{M} \varphi e^{h} f_{K}\langle\nabla h, \nabla f\rangle d \mu_{s}\right) d s \\
& -\int_{\varepsilon}^{\eta} e^{-\beta s}\left(\int_{M} \varphi^{2} e^{h} f_{K} \frac{\partial f}{\partial s} d \mu_{s}\right) d s+\alpha_{0} \int_{\varepsilon}^{\eta} e^{-\beta s}\left(\int_{M} \varphi^{2} e^{h} f_{K} f d \mu_{s}\right) d s \\
& +\alpha_{1} \int_{\varepsilon}^{\eta} e^{-\beta_{s}}\left(\int_{M} \varphi^{2} e^{h} f_{K}|\nabla f| d \mu_{s}\right) d s \\
= & \mathrm{I}+\mathrm{II}+\mathrm{III}+\mathrm{IV}+\mathrm{V}+\mathrm{VI} .
\end{aligned}
$$

We compute

$$
\mathrm{I}=-\int_{\varepsilon}^{\eta} e^{-\beta s}\left(\int_{M} \varphi^{2} e^{h}\left|\nabla f_{K}\right|^{2} d \mu\right) d s
$$

Using Schwarz' inequality we obtain

$$
\begin{aligned}
& \mathrm{II} \leqq \frac{1}{4} \int_{\varepsilon}^{\eta} e^{-\beta s}\left(\int_{M} \varphi^{2} e^{h}|\nabla f|^{2} d \mu_{s}\right) d s+\int_{\varepsilon}^{\eta} e^{-\beta s}\left(\int_{M} \varphi^{2} e^{h}|\nabla h|^{2} f_{K}^{2} d \mu_{s}\right) d s \\
& \mathrm{II} \leqq \frac{1}{2} \int_{\varepsilon}^{\eta} e^{-\beta s}\left(\int_{M} \varphi^{2} e^{h}|\nabla f|^{2} d \mu_{s}\right) d s+2 \int_{\varepsilon}^{\eta} e^{-\beta s}\left(\int_{M} e^{h}|\nabla h|^{2} f_{K}^{2} d \mu_{s}\right) d s .
\end{aligned}
$$

To estimate the terms IV + V observe that as in [16]

$$
-e^{h} f_{K} \frac{\partial f}{\partial s} \leqq-e^{h} f_{K} \frac{\partial f_{K}}{\partial s}+\frac{\partial}{\partial s}\left\{e^{h} f_{K}\left(f_{K}-f\right)\right\}
$$

whenever $\frac{\partial f_{K}}{\partial s}$ exists. Since $f_{K}$ is uniformly Lipschitz continuous on compact subsets of $M^{n} \times[0, T]$ we derive

$$
\begin{aligned}
\mathrm{IV}+\mathrm{V} \leqq & -\int_{\varepsilon}^{\eta} e^{-\beta s}\left(\int_{M} \varphi^{2} e^{h} f_{K} \frac{\partial f_{K}}{\partial s} d \mu_{s}\right) d s+\int_{\varepsilon}^{\eta} e^{-\beta s}\left(\int_{M} \varphi^{2} \frac{\partial}{\partial s}\left\{e^{h} f_{K}\left(f_{K}-f\right)\right\} d \mu_{s}\right) d s \\
& +\alpha_{0} \int_{\varepsilon}^{\eta} e^{-\beta s}\left(\int_{M} \varphi^{2} e^{h} f_{K} f d \mu_{s}\right) d s .
\end{aligned}
$$


Now observe that

$$
\left|\frac{d}{d s}\left(d \mu_{s}\right)\right| \leqq n \alpha_{3} \cdot d \mu_{s}
$$

in view of condition (iv).

Therefore,

$$
\begin{aligned}
\mathrm{IV} \leqq & -\frac{1}{2} \int_{\varepsilon}^{\eta} e^{-\beta s}\left(\int_{M} \varphi^{2} e^{h} \frac{\partial}{\partial s}\left(f_{K}^{2}\right) d \mu_{s}\right) d s+\int_{\varepsilon}^{\eta} \frac{\partial}{\partial s}\left[e^{-\beta s} \int_{M} \varphi^{2} e^{h} f_{K}\left(f_{K}-f\right) d \mu_{s}\right] d s \\
& +\beta \int_{\varepsilon}^{\eta} e^{-\beta s}\left(\int_{M} \varphi^{2} e^{h} f_{K}\left(f_{K}-f\right) d \mu_{s}\right) d s-n \alpha_{3} \int_{\varepsilon}^{\eta} e^{-\beta s}\left(\int_{M} \varphi^{2} e^{h} f_{K}\left(f_{K}-f\right) d \mu_{s}\right) d s \\
& +\alpha_{0} \int_{\varepsilon}^{\eta} e^{-\beta s}\left(\int_{M} \varphi^{2} e^{h} f_{K} f d \mu_{s}\right) d s .
\end{aligned}
$$

Choosing now $\beta \geqq 2 n \alpha_{3}+4 \alpha_{0}$ and observing that $f_{K}\left(f_{K}-f\right) \leqq 0$ we derive

$$
\begin{aligned}
\text { IV }= & -\left.\frac{1}{2} e^{-\beta s} \int_{M} \varphi^{2} e^{h} f_{k}^{2} d \mu_{s}\right|_{s=\eta}+\left.\frac{1}{2} e^{-\beta s} \int_{M} \varphi^{2} e^{h} f_{k}^{2} d \mu_{s}\right|_{s=\varepsilon} \\
& +\frac{1}{2} \int_{\varepsilon}^{\eta} e^{-\beta s}\left(\int_{M} \varphi^{2} e^{h} f_{K}^{2} \frac{\partial h}{\partial s} d \mu_{s}\right) d s-\frac{1}{4} \beta \int_{\varepsilon}^{\eta} e^{-\beta s}\left(\int_{M} \varphi^{2} e^{h} f_{K}^{2} d \mu_{s}\right) d s \\
& +\left.e^{-\beta s} \int_{M} \varphi^{2} e^{h} f_{K}\left(f_{K}-f\right) d \mu_{s}\right|_{s=\eta}-\left.e^{-\beta s} \int_{M} \varphi^{2} e^{h} f_{K}\left(f_{K}-f\right) d \mu_{s}\right|_{s=\varepsilon}
\end{aligned}
$$

The integral VI can be estimated by

$$
\mathrm{VI} \leqq \frac{1}{4} \int_{\varepsilon}^{\eta} e^{-\beta s}\left(\int_{M} \varphi^{2} e^{h}|\nabla f|^{2} d \mu_{s}\right) d s+\alpha_{1}^{2} \int_{\varepsilon}^{\eta} e^{-\beta s}\left(\int_{M} \varphi^{2} e^{h} f_{K}^{2} d \mu_{s}\right) d s
$$

Combining the estimates for I-VI, using (29) and choosing $\beta \geqq \max \left(2 n \alpha_{3}\right.$ $\left.+4 \alpha_{0}, \alpha_{1}^{2}\right)$ we conclude for $\varepsilon \rightarrow 0$ that

$$
\begin{aligned}
0 \leqq & -\int_{0}^{\eta} e^{-\beta s}\left(\int_{M} \varphi^{2} e^{h}\left|\nabla f_{K}\right|^{2} d \mu_{s}\right) d s+\int_{0}^{\eta} e^{-\beta s}\left(\int_{M} \varphi^{2} e^{h}|\nabla f|^{2} d \mu_{s}\right) d s \\
& +2 \int_{0}^{\eta} e^{-\beta s}\left(\int_{M} e^{h}|\nabla \varphi|^{2} f_{K}^{2} d \mu_{s}\right) d s-\left.\frac{1}{2} e^{-\beta s} \int_{M} \varphi^{2} e^{h} f_{K}^{2} d \mu_{s}\right|_{s=\eta} .
\end{aligned}
$$

Here we also used that $f_{K}\left(f_{K}-f\right) \leqq 0$ and $f_{K} \equiv 0$ at $s=0$. For $R>0$ let $0 \leqq \varphi \leqq 1$ satisfy $\varphi \equiv 1$ on $B_{R}^{0}(p), \varphi \equiv 0$ outside $B_{R+1}^{0}(p)$ and $|\nabla \varphi|^{0} \leqq 2$. We then conclude in view of (28)

$$
\begin{aligned}
\left.\frac{1}{2} \int_{B_{R}^{0}(p)} e^{h} f_{K}^{2} d \mu_{s}\right|_{s=\eta} \cdot e^{-\beta \eta} \leqq & \int_{0}^{\eta} e^{-\beta s}\left(\int_{B_{R+1}^{0}(p)} e^{h}\left(|\nabla f|^{2}-\left|\nabla f_{K}\right|^{2}\right) d \mu_{s}\right) d s \\
& +2 \alpha_{4} \int_{0}^{\eta} e^{-\beta s}\left(\int_{B_{R+1}^{0}(p) \backslash B_{R}^{0}(p)} e^{h} f_{K}^{2} d \mu_{s}\right) d s .
\end{aligned}
$$


Since $0<\eta<\min \left(\frac{1}{64 K}, \frac{1}{32 \alpha_{2}}\right)$ we infer $h(y, s) \leqq-2 k r_{s}^{2}(p, y)$ and $h(y, s) \leqq$ $-\alpha_{2} r_{s}^{2}(p, y)$ for all $0<s<\eta$. Since $f_{K}^{2} \leqq K^{2}$ we obtain from the volume growth assumptions on $M^{n}$ that the second term on the RHS tends to zero as $R \rightarrow \infty$. Finally, since $0 \leqq|\nabla f|^{2}-\left|\nabla f_{K}\right|^{2} \leqq|\nabla f|^{2}$ we derive for $R \rightarrow \infty$

$$
\left.\frac{1}{2} \int_{M} e^{h} f_{K}^{2} d \mu_{s}\right|_{s=\eta} \cdot e^{-\beta \eta} \leqq \int_{0}^{\eta} e^{-\beta s}\left(\int_{M} e^{h}\left(|\nabla f|^{2}-\left|\nabla f_{K}\right|^{2}\right) d \mu_{s}\right) d s
$$

in view of assumption (iii).

Now let $K \rightarrow \infty$. Then $f_{K}^{2} \rightarrow(\max (f, 0))^{2}$ and for all $s,\left|\nabla f_{K}\right|^{2} \rightarrow|\nabla f|^{2}$. Using again assumption (iii) we finally conclude

$$
\left.\frac{1}{2} \int_{M} e^{h}(\max (f, 0))^{2} d \mu_{s}\right|_{s=\eta} \leqq 0,
$$

where $0<\eta<\min \left(T, \frac{1}{64 K}, \frac{1}{32 \alpha_{2}}\right)$. By means of an inductive argument we arrive at $f \leqq 0$ everywhere in $M^{n} \times[0, T]$.

4.4 Corollary. Let $\mathbf{F}_{0}=M^{n} \rightarrow R^{n+1}$ be a smooth isometric immersion of a complete hypersurface with bounded $C^{2, \alpha}$-norm. Suppose $M_{0}=\mathbf{F}_{0}\left(M^{n}\right)$ has nonnegative mean curvature. Then the smooth solution of $(1), M_{t}=\mathbf{F}_{t}\left(M^{n}\right)$, has nonnegative mean curvature for $t \in[0, T]$ where $T$ depends on $n$ and the initial curvature bound.

Proof. Since $M_{0}$ has bounded curvature, $\sup |A| \leqq c_{0}$, it satisfies a uniform local Lipschitz condition as in Sect. 4. Using the gradient estimates in Theorem 2.1 we infer that $v$ stays bounded on some small time interval $[0, T]$ depending on $c_{0}$. By a simple modification of the arguments in the proof of Theorem 3.1 we can bound $\sup |A|$ on $[0, T]$ in terms of $c_{0}$ again. Since $\operatorname{Ric}_{M_{t}} \geqq-2|A|^{2}$ the uniform volume growth condition of Theorem 4.3 holds for $t \in[0, T]$ as we have the uniform bound on $|A|$ on this time interval. In view of the $C^{2, a}$ initial data and parabolic regularity theory we obtain the estimate

$$
\sup _{M_{t}} t^{1-\alpha}|\nabla H|^{2} \leqq c
$$

where $c$ depends on $n, T, c_{0}$ and the $C^{2, \alpha}$-norm of $M_{0}$.

This and the volume growth condition then imply the validity of condition (iii) in Theorem 4.3. Moreover (iv) holds in view of the equation

$$
\frac{d}{d t} g_{i j}=-2 H h_{i j}
$$

derived in [8]. 
In view of Lemma 1.1 (iv) and the curvature bound the mean curvature $H$ satisfies the conditions of Theorem 4.3 with $b=|A|^{2}$ for $t \in[0, T]$. This implies the result.

4.5 Remark. In a forthcoming paper Theorem 4.3 will be applied to spacelike slices moving by mean curvature in asymptotically flat spacetimes.

\section{Flow for entire graphs of arbitrary growth}

In [4] the authors established longtime existence for the initial value problem corresponding to (1) in the class of linearly growing graphs. In this section we use the interior estimates of Sect. 3 in conjunction with a spherical barrier argument to show that (1) admits a smooth solution for all $t>0$ in the class of entire graphs. In particular, no smoothness or growth assumptions have to imposed on the initial graph.

5.1 Theorem. Let $M_{0} \equiv \mathbf{F}_{0}\left(R^{n}\right)$ be a locally Lipschitz continuous entire graph over $R^{n}$. Then the initial value problem (1) has a smooth solution $M_{t}=\mathbf{F}_{t}\left(R^{n}\right)$ for all $t>0$. Moreover, each $M_{t}$ is an entire graph over $R^{n}$.

5.2 Remark. (i) In view of the equivalence of solutions of (1) and (2) discussed in Sect. 1. Theorem 5.1 also establishes the existence of a smooth solution of the initial value problem

$$
\begin{aligned}
& \frac{d}{d t} w=\sqrt{1+|D w|^{2}} \operatorname{div}\left(\frac{D w}{\sqrt{1+|D w|^{2}}}\right) \text { in } R^{n} \times(0, \infty) \\
& w(\cdot, 0))=w_{0} \quad \text { in } R^{n}
\end{aligned}
$$

assuming that $w_{0}: R^{n} \rightarrow R$ is merely locally Lipschitz continuous. Moreover, the special form of the curvature estimate in Corollary 3.2 (ii) in conjunction with the gradient estimate in Theorem 2.3 yields that at $t=0$ the solution $w$ is Hölder continuous in time with Hölder exponent $\alpha=\frac{1}{2}$.

(ii) Note that in contrast to the above result a solution of the ordinary heat equation becomes unbounded in finite time, unless certain growth conditions for $|y| \rightarrow \infty$ are imposed on the initial data.

Proof of Theorem 5.1. Assume first $M_{0}=\mathbf{F}_{0}\left(R^{n}\right)=$ graph $w_{0}$ for $w_{0} \in C^{2, \alpha}\left(R^{n}\right)$, $\alpha>0$.

Using Theorem 2.1 of [12] as well as the tangential diffeomorphisms defined in Sect. 1 we infer that for any $R>0$ the boundary value problem

$$
\begin{aligned}
\frac{d}{d t} \mathbf{F}(y, t) & =\mathbf{H}(y, t), & & (y, t) \in B_{R}(0) \times(0, \infty) \\
\mathbf{F}(y, 0) & =\mathbf{F}(y), & y & \in B_{R}(0) \\
\mathbf{F}(y, t) & =\mathbf{F}(y), & (y, t) & \in \partial B_{R}(0) \times[0, \infty)
\end{aligned}
$$

admits a smooth solution $M_{t}^{R}=F_{t}^{R}\left(\bar{B}_{R}(0)\right)$ which for each $t>0$ can be written as a graph over $\bar{B}_{R}(0) \subset R^{n}$. 
Let now $T>0$ be fixed and $\Omega \subset R^{n}$ be compact. Choose $R_{0}>0$ s.t. $\Omega \subset B_{R_{0}}(0)$ and define $R_{1}=\sqrt{4 R_{0}^{2}+2 n T}$.

We also define $a^{+}=\left(0, \sup w_{0}+R_{1}+1\right) \in R^{n+1}$. Then we have for $R>R_{1}$ $B_{R_{1}}(0)$

$$
M_{0}^{R} \cap B_{R_{1}}^{n+1}\left(a^{+}\right)=\emptyset
$$

where $B_{R_{1}}^{n+1}$ denotes a ball in $R^{n+1}$. Furthermore

$$
\partial M_{t}^{R} \cap B_{R_{1}}^{n+1}\left(a^{+}\right)=\emptyset
$$

for $t \geqq 0$. Using Theorem 3.7 of [1], or Lemma 3.2 of [11], we infer

$$
M_{t}^{R} \cap B_{2 R_{0}}^{n+1}\left(a^{+}\right)=\emptyset
$$

for $t \in[0, T]$.

Using a similar argument for $B_{R_{1}}^{n+1}\left(a^{-}\right)$, where $a^{-}=\left(0, \inf _{B_{R_{1}}(0)} w_{0}-R_{1}-1\right)$ $\in R^{n+1}$ we conclude that the height function $w_{R}(\cdot, t)$ of $M_{t}^{R}$ satisfies

$$
\sup _{B_{2 R_{0}} \times[0, T]}\left|w_{R}\right| \leqq c_{0}
$$

where $c_{0}=c_{0}\left(n, R_{0}, T\right.$, sup $\left.w_{0}\right)$ is independent of $R$.

$$
{ }_{B_{R_{1}}}(0)
$$

Applying the gradient estimate of Theorem 2.3 we obtain

$$
\sup _{B_{3 / 2 R_{0}}(0) \times[0, T]}\left|D w_{R}\right| \leqq c_{1}
$$

where $c_{1}=c_{1}\left(n, R_{0}, c_{0}, \sup _{\mathbf{B}_{2 \mathbf{R}_{0}}(0)}\left|D w_{0}\right|\right)$.

From Corollary 3.5 (ii) we then conclude for any integer $m \geqq 0$

$$
\sup _{B_{R_{0}}(0) \times[0 . T]}\left|D^{m} w_{R}\right| \leqq c_{m}
$$

where $c_{m}=c_{m}\left(m, n, R_{0}, c_{0}, c_{1}\right)$.

We can therefore select a sequence of solutions $\left(w_{R_{k}}\right)$ for $R_{k} \rightarrow \infty\left(R_{k}>R_{1}\right.$ for any $k \geqq 2$ ) s.t. $w_{R_{k}} \rightarrow w$ in $C^{\infty}$ uniformly on $\Omega \times[0, T]$. Since $\Omega$ and $T>0$ were arbitrary this establishes the existence of a family of entire graphs $M_{t}$ $=$ graph $w(\cdot, t)$ solving (1) where $w \in C^{\infty}\left(R^{n} \times(0, \infty)\right)$. As the second and higher order derivative estimates for $w$ on each compact subset of $R^{n}$ depend only on the initial height and gradient on a slightly larger subset, an approximation argument yields a smooth solution of (1) also for locally Lipschitz initial data.

\section{References}

1. Brakke, K.A.: The Motion of a Surface by its Mean Curvature. (Math. Notes) Princeton, NJ: Princeton University Press 1978

2. Caffarelli, L., Nirenberg, L., Spruck, J.: On a form of Bernstein's Theorem. (Preprint)

3. Ecker, K.: Estimates for evolutionary surfaces of prescribed mean curvature. Math. Z.

180, 179-192(1982) 
4. Ecker, K., Huisken, G.: Mean curvature evolution of entire graphs. Ann. Math. 130, 453-471 (1989)

5. Ecker, K., Huisken, G.: A Bernstein result for minimal graphs of controlled growth. J. Differ. Geom. 31, 397-400 (1990)

6. Gerhardt, C.: Evolutionary surfaces of prescribed mean curvature. J Differ. Equations 36, 139-172 (1980)

7. Grayson, M.: The heat equation shrinks embedded plane curves to round points. J. Differ. Geom. 26, 285-314 (1987)

8. Hamilton, R.S.: Three-manifolds with positive Ricci curvature. J. Differ. Geom. 17, 255-306 (1982)

9. Hamilton, R.S.: Heat Equations in Geometry. Honolulu, Hawaii (Lecture Notes) 1989

10. Huisken, G.: Flow by mean curvature of convex surfaces into spheres. J. Differ. Geom. 20, 237-266 (1984)

11. Huisken, G.: Contracting convex hypersurfaces in Riemannian manifolds by their mean curvature. Invent. Math. 84, 463-480 (1986)

12. Huisken, G.: Non-parametric mean curvature evolution with boundary conditions. J. Differ. Equations 77, $369 \cdots 378$ (1989)

13. Huisken, G.: Asymptotic behaviour for singularities of the mean curvature flow. J. Differ. Geom. 31, 285-299 (1990)

14. Korevaar, N.: An easy proof of the interior gradient bound for solutions to the prescribed mean curvature equation. Proc. Symp. Pure Math. 45, Part 2, 81 -89 (1986)

15. Ladyzhenskaya, O.A., Solonnikov, V.A., Uraltseva, N.N.: Linear and Quasilinear Equations of Parabolic Type. Transl., II. Ser., Am. Math. Soc. 23 (1968)

16. Liao, G.G., Tam, L.-F.: On the heat equation for harmonic maps from non-compact manifolds. (Preprint)

17. Shi, W.-X.: Deforming the metric on complete Riemannian manifolds. J. Differ. Geom. 30, 223-301 (1989)

18. Angenent, S.: Parabolic equations for curves on surfaces (I). Curves with p-integrale curvature, preprint Univ. of Wisconsin, 1988 